Rapid Communications

\title{
Stabilization of the Spark-discharge Point on a Sample Surface by Laser Irradiation for Steel Analysis
}

\author{
Hideyuki Matsuta, ${ }^{* \dagger}$ Kuniyuki KitagaWa, ${ }^{* *}$ and Kazuaki Wagatsuma* \\ *Institute for Materials Research, Tohoku University, Katahira 2-1-1, Sendai 980-8577, Japan \\ **Ecotopia Science Institute, Nagoya University, Furo-cho, Chikusa-ku, Nagoya 464-8603, Japan
}

\begin{abstract}
A combined technique with laser irradiation is suggested to control spark discharge for analytical use, having a unique feature that firing points of the spark discharge can be fixed by laser irradiation. Because the spark discharge easily initiates at particular surface sites, such as non-metallic inclusions, called selective discharge, the concentration of some elements sometimes deviates from their average one in spark discharge optical emission spectrometry. Therefore, stabilization of firing points on a sample surface could improve the analytical precision.
\end{abstract}

(Received August 4, 2006; Accepted August 21, 2006)

In the steel-making industry, spark discharge-optical emission spectrometry (OES) is widely used as an analytical means for process control because of its high sensitivity and rapid analytical features. However, the analytical precision of the spark discharge-OES is greatly affected by the sample surface conditions, since spark discharges initiate easily at surface sites, such as non-metallic inclusions (e.g. $\left.\mathrm{Al}_{2} \mathrm{O}_{3}\right)$, surface cracks, and grain boundaries. As a result, the determined concentration of some elements sometimes deviates from their averaged one in the sample. Therefore, a time-consuming pretreatment of the sample, such as surface polishing, is needed to obtain better analytical results. If spark discharges could be initiated at a controlled position regardless of the surface conditions, the analytical precision of the spark discharge-OES would be greatly improved. In this report, a combined technique with laser irradiation is suggested to control spark discharges for analytical use.

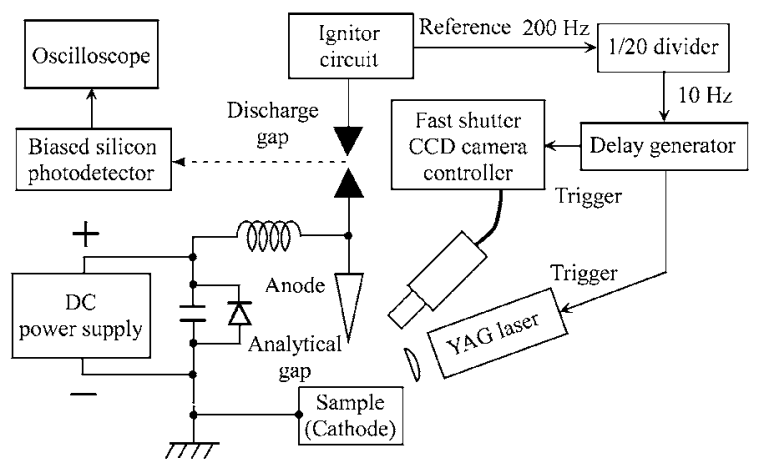

Fig. 1 Schematic diagram of the apparatus.

$\dagger$ To whom correspondence should be addressed. E-mail: matsuta@imr.tohoku.ac.jp

\section{Experimental}

A schematic diagram of the apparatus and the details of the experimental set-up are shown in Fig. 1 and Table 1. All of the experiments were performed in the open air. The repetition rate of spark discharges was predetermined at $200 \mathrm{~Hz}$. The starting of each spark discharge was determined by monitoring pulsed light at the discharge gap of an ignitor circuit with a biased silicon photodetector. The time difference between the spark firing and the clock signal for regulating the ignitor circuit was observed to be about $1 \mathrm{~ms}$. The timing of laser irradiation and the gate timing of a CCD (charge coupled device) camera were determined by this clock signal as well as the delay time. The maximum repetition rate of the Nd:YAG laser and the frame rate of the CCD camera were also below $10 \mathrm{~Hz}$; therefore, the TTL (transistor transistor logic)-level clock signal of the ignitor $(200 \mathrm{~Hz})$ was divided into $1 / 20$ by using a laboratory-made frequency divider to generate a trigger signal of $10 \mathrm{~Hz}$. The laser $(532 \mathrm{~nm})$ was focused tightly on the sample surface and the angle of incidence was about 45 degrees from the normal.

Table 1 Components of instrumentation

\begin{tabular}{ll}
\hline \multicolumn{1}{c}{$\begin{array}{c}\text { Spark discharge optical } \\
\text { emission source }\end{array}$} & $\begin{array}{c}\text { Shimadzu HPSG-500 (optical } \\
\text { emission source of PDA-5500 II) }\end{array}$ \\
\hline Nd:YAG laser & $\begin{array}{c}\text { Lotis TII LS-2135 } \\
\text { Wave length: } 532 \mathrm{~nm} \text { (SHG) } \\
\text { Pulse duration: } 10 \mathrm{~ns}\end{array}$ \\
& Pulse energy: $3-20 \mathrm{~mJ}$ \\
Focusing lens & Plano-convex, $f=100 \mathrm{~mm}$ \\
Delay generator & Stanford Research Systems DG535 \\
Fast shutter CCD camera & PCO imaging SensiCam 370KD-II \\
& Exposure time: $1 \mu \mathrm{s}$ \\
Camera lens & Nikon UV-Nikkor \\
Biased silicon photodetector & Electro-Optics Technology ET-2030 \\
Sample & Fe plate (99.99\%, purity) \\
\hline
\end{tabular}


Table 2 Determination of the operating parameters for stable spark discharge

\begin{tabular}{cccc}
\hline \multicolumn{1}{c}{ Parameter } & \multicolumn{3}{c}{ Combination of parameters } \\
\hline $\begin{array}{c}\text { Electrode spacing } \\
\text { Pulse energy of } \\
\text { laser (532 nm) }\end{array}$ & $17 \mathrm{~mJ}$ (fix) & $>9 \mathrm{~mm}$ (fix) & $3 \mathrm{~mm}$ (fix) \\
$\begin{array}{c}\text { Delay time } \\
\text { laser firing }\end{array}$ & $-6 \mu \mathrm{s}$ (fix) & $-6 \mu \mathrm{s}$ (fix) & From -6 to $-200 \mu \mathrm{s}$ \\
\hline
\end{tabular}

a. In this paper, "stable" means that a misfiring of the spark discharges seldom occurs.

b. Delay time was measured from the onset time of the high voltage applied by the ignitor circuit. Minus sign shows advancement.

\section{Results and Discussion}

When a tungsten stylus having a round-shaped tip and an iron plate were used as the counter and the sample electrodes, it was very difficult to induce spark discharges between these electrodes having distances of a few $\mathrm{mm}$ in air, and a closer spacing of less than $0.7 \mathrm{~mm}$ was required to generate the spark discharge under our experimental conditions. It has been reported that a laser-induced plasma can work as a trigger for inducing a spark discharge. ${ }^{1,2}$ We employed this combined technique and investigated three experimental parameters: the electrode spacing, the energy of a pulsed laser and the delay time of laser irradiation, to minimize any misfiring of the spark discharge. The condition under which the misfiring seldom occurs is called "stable" in this paper. A parameter set for the optimum condition is indicated in Table 2. By introducing laser irradiation, the electrode distance could be extended up to 3.5 $\mathrm{mm}$ while keeping stable spark discharges. We also found an interesting phenomenon regarding the timing between the spark discharge and the laser irradiation. Whereas stable spark discharges occur when a laser-induced plasma has been generated before a high voltage is applied to the electrodes (time difference of 6-200 $\mu \mathrm{s}$ ), spark discharges cannot be induced when the laser irradiation occurs just at the moment when, or after, a high voltage is applied. In the case where a high voltage for spark discharges is applied at delay times of 6 and $50 \mu \mathrm{s}$ after laser irradiation, time-resolved images of the spark discharges were taken, and are shown in Figs. 2 and 3.

A direct-current discharge is initially induced by electrons emitted from the cathode surface. Because a large number of electrons are produced at the surface position where laser breakdown occurs, the following spark discharge can be easily developed just at the breakdown position, as shown in Fig. 2. It is known that the inner pressure of a laser-induced plasma reaches up to several tens of MPa immediately after breakdown, and then decreases as the time proceed. ${ }^{3}$ Because the mean-free path of the electrons is very small under such high-pressure conditions, the electrons would not work as a seed for the subsequent spark discharge. However, the electrons could run outside as the inner pressure of the laser-induced plasma decreases. Figure 2 implies that a delay time of $6 \mu$ s or more is needed to obtain appropriate pressures so that the electric field of the spark discharge can draw electrons from the laserinduced plasma. It is also found from these figures that the laser-induced plasma disappears within $20 \mu \mathrm{s}$ after laser irradiation. Even when a high voltage is applied to the electrodes after the laser-induced plasma has disappeared, spark discharges without misfiring are observed, as shown in Fig. 3. This effect is probably because the temperature of the burning

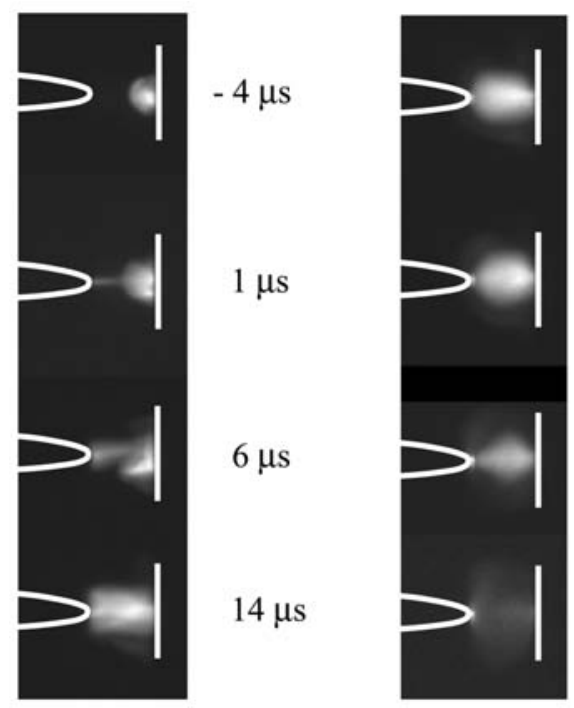

$24 \mu \mathrm{s}$

$34 \mu \mathrm{s}$

$54 \mu \mathrm{s}$

$74 \mu \mathrm{s}$

Fig. 2 Time-resolved images of the spark discharge. A laserinduced plasma was created $6 \mu$ s before applying a high voltage to the electrodes. The opening time of the shutter described in the figure was measured from the onset time of a high voltage applied to the electrodes. The exposure time of a fast-shutter CCD camera was $1 \mu \mathrm{s}$.
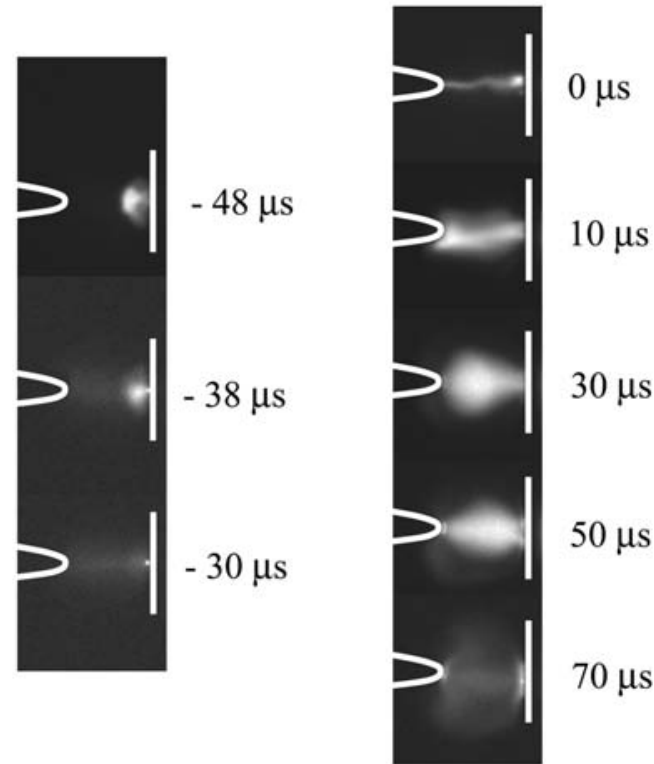

Fig. 3 Time-resolved images of the spark discharge. A laserinduced plasma was created $50 \mu$ s before applying a high voltage to the electrodes. The opening time of the shutter described in the figure was measured from the onset time of a high voltage applied to the electrodes. The exposure time of the fast-shutter CCD camera was $1 \mu \mathrm{s}$.

position is sufficiently high to emit thermo-electrons to work as a seed for the spark discharge. It is understood from Table 2 that such thermo-electrons remain for $200 \mu \mathrm{s}$ after laser irradiation.

If this type of electron emission can control the following spark discharge, the spark channels between the electrodes can be also controlled by the laser shots, even though the electrode distance is separated out of the range for a stable spark discharge to occur. In order to confirm this, we moved a tungsten stylus electrode to within $\pm 2 \mathrm{~mm}$ horizontally from the breakdown position of the laser-induced plasma (delay time of 


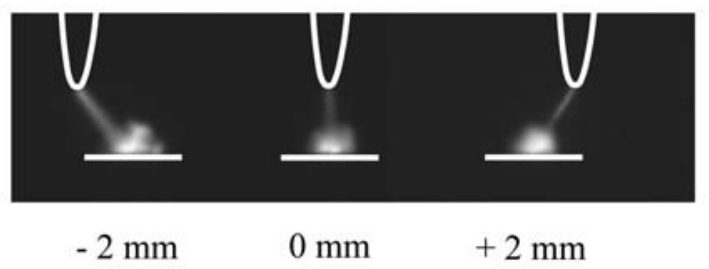

Fig. 4 Time-resolved images of the spark discharge. The stylus anode was moved to $0, \pm 2 \mathrm{~mm}$ horizontally from the breakdown position. A high voltage was applied to the electrodes $6 \mu \mathrm{s}$ after creating a laser-induced plasma.

$-6 \mu \mathrm{s})$. Time-resolved images are illustrated in Fig. 4. The spark channel could be maintained between the stylus electrode and the breakdown position regardless of the length of the spark channels.

It is found that the initiation site of a spark discharge can be controlled by laser irradiation. Since sample atoms vaporize at this position during spark discharge, the precision of spark discharge-OES is expected to be improved by the present method, regardless of the surface conditons of the sample, which greatly affect the starting point of the spark discharge.

\section{Acknowledgements}

This work was performed under the Inter-University Cooperative Research Program of the Institute for Materials Research, Tohoku University. A part of this work was supported by a Grant-in-Aid from Japan Society for the Promotion of Science (No. 17560649).

\section{References}

1. K. Laqua, "Analytical Laser Spectroscopy", ed. N. Omenetto, 1979, Chap. 3, Wiley Interscience, New York.

2. O. Ayed Nassef and H. E. Elsayed-Ali, Spectrochim. Acta, Part B, 2005, 60, 1564.

3. R. G. Root, "Laser-Induced Plasma and Applications", ed. L. J. Radziemsky and D. A. Cremers, 1989, Chap. 2, Marcel Dekker, New York. 